\title{
Biomarkers associated with coronavirus disease-19 disease progression in a Mexican hospital
}

\author{
Raquel Gerson-Cwilich ${ }^{1 *}$, Daniela Shveid-Gerson ${ }^{1}$, Alejandro Noguez-Ramos ${ }^{1}$, Lorena López-Zepeda ${ }^{1}$, \\ Benigno E. Rodríguez-Soto ${ }^{1}$, Diana A. Villegas-Osorno ${ }^{1}$, Benjamín Valente-Acosta ${ }^{2,3}$, \\ Javier García-Guerrero ${ }^{2,3}$, and Francisco Moreno-Sánchez ${ }^{2,3}$ \\ ${ }^{1}$ Department of Medical Oncology; ${ }^{2}$ Department of Infectious Diseases; ${ }^{3}$ Department of Internal Medicine. The ABC Medical Center IAP, Mexico \\ City, Mexico
}

\begin{abstract}
Introduction: The Coronavirus disease (COVID-19) pandemic is a worldwide challenge. There are few useful tools to predict patient outcomes. Identification of biomarkers able to predict progression of the disease could improve the treatment of these patients. Objective: The objective of the study was to identify biomarkers of disease progression among patients with severe COVID-19 pneumonia. Materials and methods: A retrospective cohort study was conducted among severe COVID-19 pneumonia patients hospitalized in the American British Cowdray Medical Center in Mexico City. Disease progression was defined as use of vasoactive amines, need of non-invasive or invasive mechanical ventilation or death. Studied biomarkers included neutrophilllymphocyte index, lymphocyte/platelet Ratio, C reactive protein, procalcitonin, D Dimer, lactic dehydrogenase (LDH), ferritin, 25-OH-Vitamin D, and interleukin 6. Results: We report 46 patients with severe COVID-19 pneumonia. Mean age was 51 years, the majority of whom $30(65 \%)$ male. Median hospitalization was 9 days. $23(50 \%)$ of patients presented disease progression. Ferritin and LDH were strongly associated with disease progression among our cohort. In addition, age was associated with worst prognosis with a relative risk $4.5(1.2-16.9, p=0.003)$. Conclusions: Age, ferritin, and LDH were associated with disease progression among patients with severe COVID-19 pneumonia.
\end{abstract}

Key words: Severe acute respiratory syndrome coronavirus 2. Coronavirus disease-19. Biomarkers. Lactic dehydrogenase. Ferritin. Mexico.

\section{Introduction}

In the final months of 2019, a new type of coronavirus was associated to the development of pneumonia in the city of Wuhan, China ${ }^{1}$. Its appearance was traced back to the consumption of food (a water snake) at an open-air market in this city. A short time after this discovery was made, a beta-coronavirus, was isolated as the etiologic virus, causing what is now known as severe acute respiratory syndrome coronavirus 2 (SARS - COV 2). The evidence presented on the isolation of the virus included bronquio-alveolar lavage specimens, which were, in turn, submitted to indirect genomic sequencing by polymerase chain reaction (PCR) and viral culture. The dissemination of this new agent was quick to originate an epidemic in a matter of weeks. By February 2020, cases continued to increase, prompting the World Health Organization to declare it a pandemic in March 20201,2. Until now, there have been more than 10 million cases have been confirmed worldwide by the World Health Organization, including
Correspondence:

*Raquel Gerson-Cwilich

E-mail: rgerson1@yahoo.com DOI: 10.24875/HGMX.20000 0185-1063/@ 2020 Sociedad Médica del Hospital General de Mexico. 
the first cases reported in China at the end of 2019. The infection has now spread through all continents except Antarctica. The accumulated incidence pertaining to each place where the pandemic propagates depends on certain factors, which include demographic density, the detection, report and application of tests, and strategies to reduce the spread of the virus ${ }^{1,2}$.

\section{Transmission and clinical manifestations}

The primary form of contagion of SARS-COV-2 is person-to-person contact. Contact with flügge droplets and exposure to the mucosa, whether it be nasal, oral, or ocular, to agents pertaining to the airway of infected persons who cough, sneeze, or talk are the most prevalent ways of contracting the virus. In addition, contact with surfaces where the virus is present and the afterward contact with the previously stated mucosa is a noteworthy form of transmission ${ }^{1,3}$. The virus can present itself in a symptomatic or asymptomatic fashion way, which varies depending on the severity of infection. The proportion of asymptomatic patients has yet to be further studied, as it is estimated that they comprise up to $30-40 \%$ of all cases reported. In addition, it is estimated that the incubation period for this virus varies from 1 to 14 days, with a median estimated time of 5 days to develop symptoms ${ }^{4}$.

The virus may be transmitted during the incubation period. The pathophysiology of this new disease is not well known, notwithstanding, some studies suggest that the virus may form a bond with the angiotensin 2 converting enzyme, a similarity shared with the already known SARS virus. An elevated viral load has been detected in the nasal mucosa and pharynx of patients as soon as symptoms develop. It is notable to say that asymptomatic patients may have a viral load as elevated as that of patients with severe symptoms ${ }^{5,6}$. Signs and symptoms associated with this disease are similar to those of a viral pneumonia, its gravity ranging from mild to severe. Approximately $80 \%$ of patients initiate with mild disease, $14 \%$ with severe disease, and $5 \%$ of patients are in need of critical care medicine. Some reports suggest that the severity of the disease is strongly correlated with age and preexisting comorbidities. Among the most common comorbities are cardiovascular disease, diabetes mellitus, hypertension, chronic pulmonary obstructive disease, obesity, and chronic kidney disease ${ }^{4,5}$.

The most common symptoms include fever, cough, dyspnea, myalgia, and fatigue. Among the less frequent are anorexia, sputum production, confusion, rhinorrhea, headache, anosmia, loss of taste, nausea, vomiting, diffuse abdominal pain, and diarrhea ${ }^{6}$. The vast majority of patients present one or more symptoms. We observed an average of $15 \%$ presented with fever, cough and dyspnea on onset of the disease 4 .

Laboratory anomalies in hospitalized patients with SARS-COV 2 pneumonia include leucopenia, with leukocytosis in some cases, lymphopenia, elevated liver enzymes, thrombocytopenia, anemia, hypoalbuminemia, or acute renal injury. More than half of patients are evaluated with $<90 \%$ of oxygen saturation. Some studies have related the severity of the disease, both in presentation and outcomes, to the elevation of biochemical markers, such as de elevation of lactic dehydrogenase (LDH), ferritin, interleukin 6 (IL6), and D dimer $^{3,5,6}$. Some patients with severe disease have a laboratorial confirmation of an exuberant inflammatory response, comparable to a cytokine release syndrome or a cytokine storm, associated, in turn, with persistent fever and the elevation of the aforementioned biomarkers. As such, the elevation of these biomarkers has been associated with critical and even fatal disease ${ }^{5}$. The aggressive inflammatory response that occurs in this disease is intrinsically related to the damage caused in the respiratory system 7 . Thus, the severity of the disease is not something related only to the viral infection that is in intertwined with the host's response. Cytokine release in response to a sustained viral infection, specifically pro-inflammatory cytokines, may result in symptoms associated with sepsis. The attributing factor to approximately $28 \%$ of SARS-COV 2 deaths, as in these cases, uncontrolled inflammation leads to multiple organ failure, particularly renal, cardiovascular, and hepatic ${ }^{8}$.

Among known pro-inflammatory cytokines, we may find IL6, IP-10, MIP1 alfa, and beta as well as MCP, which bind in turn to monocytes, macrophages, and $T$ cells, promoting the production of gamma interferon. This creates a cycle of persistent and sustained inflammation. Pathogen-associated molecular patterns such as viral RNA and danger-associated molecular patterns, including adenosine triphosphate and DNA are detected by pattern recognition receptors, alveolar epithelial cells and alveolar macrophages. In turn, this provokes the propagation of local inflammation, involved in the increased secretion of IL6, IL10, gamma interferon, and MCP. Other mediators or inflammatory response observed in Coronavirus disease (COVID-19) are IL2, IL7, IL10, G- CSF, TNF, and CD14 + CD16 + monocytes. The regulation of the secretion of pro-inflammatory cytokines such as IL1 beta and IL8 is 
regulated by the NLRP3 inflammasome, creating reactive oxygen species and viroporins, pore forming viral proteins, which modify cellular membranes ${ }^{9}$.

As previously commented in clinical and laboratorial terms, $\mathrm{C}$ reactive protein (CRP), procalcitonin, erythrocyte sedimentation rate, serum amyloid protein, IL6, ferritin, and fibrinogen have been evaluated in the development of infection by COVID - 19 and as markers of systemic inflammation and disease severity and progression. Low levels of procalcitonin with high levels of CPR are correlated in this entity, as well as higher levels of IL6 were reported in people who died from this disease $^{10}$. Nonetheless, PCT an procalcitonin levels may be higher in patients with severe SARS-COV 2 infection. In relation to this, CD14 + CD 16+ monocytes activated by granulocyte - marcrophage colony stimulating factor (GM - CSF) secrete more IL6 than other pro-inflammatory markers. There exists a heterogeneous gamma of laboratory results, as specific levels may depend on secondary infections, variability in laboratory levels, and differences with those reported worldwide, whether it be technique or standard cutoff value, as well as bias in publications depending on the county or region affected. Another marker for disregulation of the immune system, which permits the development of viral hyper-inflammation is the neutrophil/lymphocyte Ratio (NLR) and the lymphocyte/platelet Ratio (LPR). It is theorized that these indexes may help predict clinical severity in patients with COVID 19, specifically, expecting NLR levels to increase significantly while LPR levels reduce, reflecting, in turn, an acute inflammatory process and possibly a worse prognosis ${ }^{11}$.

Evidence exists which suggests that an elevation of LDH above 350 IU may be considered an important marker for the severity of infection by COVID 19. Furthermore, a negative impact is assumed regarding the survival with patients with pneumonia. This was observed in patients who had an AH1N1 influenza pneumonia, infection by pneumocystis jirovecci and bacterial pneumonia. In the specific context of patients with SARS-COV 2 pneumonia, an increment in LDH levels has been observed as a possible predictor of tissue damage. Information is needed to establish this biomarker as a prognostic tool in patients with severe disease $e^{2,5,6}$.

\section{Materials and methods}

A retrospective cohort study was conducted including severe COVID-19 hospitalized patients in the American British Cowdray (ABC) Medical Center. The infection was confirmed by oropharyngeal RT-PCR to detect
SARS-CoV-2. All patients had severe COVID-19 disease and required supplementary oxygen. Studied biomarkers include glucose, albumin, neutrophil/ lymphocyte ratio (NLR), LPR, CRP, procalcitonin, D Dimer, LDH, ferritin, 25 - OH - Vitamin D, and IL6.

Clinical and laboratorial variables were collected on the patients' admission. Electronic medical records were assessed to evaluate disease progression, which was defined as patients who needed vasoactive amines, invasive, or non-invasive mechanical ventilation or death.

Data were analyzed using the statistical program SPSS version 22 (Chicago, IL). For variables which had a normal distribution, the mean and standard deviation were used and for free distribution variables, the median, and interquartile range. To contrast the differences which were found between the groups with or without complications, a T student test or a Mann-Whitney U-test were used for quantitative variables or normal distribution or free distribution, respectively. For qualitative variables, a Chi-square test was applied. For determination of the best cutoff value for each variable, a receiver operating characteristic (ROC) curve was used. With regard to the primary outcome, a Chi-square test and relative risk (RR) were utilized.

\section{Results}

We report 46 patients hospitalized with SARS-COV 2 severe infection confirmed by RT-PCR in the ABC Medical Center in Mexico City. The mean age was 51 years SD (16.7), the majority of whom were male $30(65 \%), 38(82 \%)$ being overweight or obese, 10 (21\%) had type 2 diabetes mellitus, and 15 (32\%) had hypertension (Table 1).

The median hospitalization was 9 days (interquartile range of 6-21 days). Patients who presented disease progression were $23(50 \%)$. Disease progression includes death of 4 patients $(8.7 \%)$, invasive mechanical ventilation in 20 patients $(43.5 \%)$, non-invasive ventilation in 12 patients (26.1\%), and use of vasoactive amines in 17 patients (37\%). The median days until death were 18. During treatment, steroids were used in $16(34 \%)$ of the patients. Other treatments used were antibiotics, antivirals, and tocilizumab. An analysis by treatment type was not performed. While contrasting the patients' basal characteristics with the presence of complications, age, and levels of LDH stand out among the studied variables (Table 2).

The variables of significant association with the primary outcome comprised of death, use of vasoactive 
Table 1. Basal characteristics of patients on entry to hospitalization

\begin{tabular}{|c|c|}
\hline Characteristics & Value \\
\hline Age & 51.5 (16.7) \\
\hline Gender & $\begin{array}{c}\text { Men } 30(65.2 \%) \\
\text { Women } 16(34.8 \%)\end{array}$ \\
\hline Cancer & $2(4.3 \%)$ \\
\hline Type 2 diabetes mellitus & $10(21.7 \%)$ \\
\hline Hypertension & 15 (32.6\%) \\
\hline Body mass index (BMI) $\left(\mathrm{Kg} / \mathrm{m}^{2}\right)$ & $27.4(25.8-30.8)$ \\
\hline Overweight or obesity BMI > $25 \mathrm{Kg} / \mathrm{m}^{2}$ & $38(82.6 \%)$ \\
\hline Glucose (mg/dL) & $111(98-129)$ \\
\hline Leukocytes $\left(\times 10^{9} / \mathrm{L}\right)$ & $7.2(4.6-11.2)$ \\
\hline Neutrophils $\left(\times 10^{9} / \mathrm{L}\right)$ & $6.1(3.9-12.2)$ \\
\hline Lymphocytes $\left(\times 10^{9} / \mathrm{L}\right)$ & $0.96(0.7-1.3)$ \\
\hline Hemoglobin $(\mathrm{g} / \mathrm{dL})$ & $14.7(13.5-16.1)$ \\
\hline Platelets $\left(10^{9} / \mathrm{L}\right)$ & $222(146-280)$ \\
\hline Lactate $(\mathrm{mmol} / \mathrm{L})$ & $1.4(1.0-1.9)$ \\
\hline Creatinine $(\mathrm{mg} / \mathrm{dL})$ & $0.9(0.7-1.0)$ \\
\hline Albumin $(\mathrm{g} / \mathrm{dL})$ & $3.6(0.4)$ \\
\hline 25-OH-Vitamine D (ng/dL) & $15.5(12.9-19.6)$ \\
\hline Lactic dehydrogenase (LDH) (UI/L) & $310(215-418)$ \\
\hline D Dimer (mcg/L) & $841(564-1384)$ \\
\hline Procalcitonin (ng/mL) & $0.2(0.09-0.63)$ \\
\hline$C$ reactive protein $(\mathrm{mg} / \mathrm{L})$ & $11.9(5.5-21.9)$ \\
\hline Ferritin (mcg/L) & $799(420-1522)$ \\
\hline IL-6 (pg/mL) & $73.8(33-202)$ \\
\hline Neutrophil/lymphocyte Ratio (NLR) & $6.7(4.2-12.7)$ \\
\hline Lymphocyte/platelet Ratio (LPR) & $221(139-319)$ \\
\hline
\end{tabular}

For normal distribution mean and standard deviation were used and for free distribution the median and interquartile range.

amines or mechanical ventilation include age, glucose, and the LDH levels on hospital admission. The cutoff levels were age above 50 years, glucose levels above $126 \mathrm{mg} / \mathrm{dL}$, and LDH levels above $410 \mathrm{UI} / \mathrm{L}$ (Table 3). ROC curves were created for each of the studied variables to determine the best cutoff point for their associations to complications registered during their hospitalization. For age, an area below the curve of $0.72(0.57-0.87, p=0.008)$ was established, for the levels of LDH, 0.70 (0.55-0.85, $p=0.01)$, and for glucose $0.81(0.68-0.93, p \leq 0.001)$ (Fig. 1).
No association was found between the primary outcome and type 2 diabetes mellitus (RR 1.57, 0.91-2.71, $p=0.28$ ) or hypertension (RR 1.59, 0.92-2.74, $p=0.20$ ).

The primary outcome was broken down and a sub-analysis was performed between each variable. Statistical significance was found between invasive mechanical ventilation in patients above 50 years of age (RR 3.93, 1.05-14.72, $p=0.01$ ), glucose levels above $126 \mathrm{mg} / \mathrm{dL}$ (RR 3.05, 1.65-5.63, $p=0.001$ ), and LDH levels above $410 \mathrm{UI} / \mathrm{L}$ (RR 2.12, 1.18-3.80, $p=0.03$ ). In addition, vasoactive amines use was associated with age above 50 years (RR 7.0, 1.02-47.75, $p=0.007$ ) and glucose levels above $126 \mathrm{mg} / \mathrm{dL}$ (RR 3.53, 1.69-7.36, $\mathrm{p}=0.002$ ).

\section{Discussion}

In this retrospective cohort study, we show the characteristics and clinical outcomes of 46 patients hospitalized in the $A B C$ Medical Center infected with COVID-19. Although many clinical characteristics are similar to those reported in the previous studies ${ }^{12}$, the fact that this has been reported in different regions of the world underlines the importance of studying the different clinical features present in our population, and with this, biomarkers which may help predict a worse outcome. It may be observed that almost the double of patients who were admitted with severe COVID-19 pneumonia were men $(65.5 \%$ vs. $34.8 \%)$, as has been noted in national statistics. This differs with reports from other countries, in which the reported incidence is similar in both genders (men 59\% and women $41 \%^{12-14}$ ), as well as male mortality, documented to be almost 2.4 times more in women, regardless of age or comorbidities $^{15}$. There has been speculation that an explanation to this phenomenon is the protective role estrogens play in severe infection, angiotensin-2 receptors, which have been described as the entry point of the virus, and the anti-inflammatory role the angiotensin-converting enzyme has. Specifically, its gene is located in the $\mathrm{X}$ chromosome, which may be correlated to the higher levels of this enzyme in women and as such, an additional protective factor ${ }^{16}$.

Among the comorbidities reports in our population, it is important to note there exists a higher proportion of patients with hypertension (32.6\%) in contrast to national statistics (19.7\%) and world statistics (6\%). Regarding type two diabetes mellitus; in our study, there was a prevalence of $21.7 \%$, once again higher in relation to national statistics $(16.5 \%)$, and world statistics $(6.3 \%)^{13,16}$. It is, therefore, interesting to note that this increase in the prevalence of comorbidities in our 
Table 2. Basal characteristics of patients on entry to hospitalization according to the presence of in-hospital complications

\begin{tabular}{|c|c|c|c|}
\hline Characteristics & With complications ( $n=23$ ) & Without complications ( $n=23$ ) & $\mathrm{p}$ value \\
\hline Age (Media) & 62 & 49 & 0.004 \\
\hline Gender (Men) & $16(69.6 \%)$ & $14(60.9 \%)$ & 0.53 \\
\hline Cancer & $2(8.7 \%)$ & 0 & 0.48 \\
\hline Type 2 diabetes mellitus & $7(30.4 \%)$ & $3(13 \%)$ & 0.28 \\
\hline Hypertension & $10(43.5 \%)$ & $5(21.7 \%)$ & 0.20 \\
\hline BMI $\left(\mathrm{kg} / \mathrm{m}^{2}\right)$ (Mean) & 27.7 & 27.7 & 1.0 \\
\hline Overweight or obesity (Mean) & $19(82.6 \%)$ & $19(82.6 \%)$ & 0.66 \\
\hline Glucose $(\mathrm{mg} / \mathrm{dL})$ & 183 & 104 & 0.14 \\
\hline Leucocytes $\left(\times 10^{9} /\right.$ L) (Mean) & 8.6 & 6.7 & 0.37 \\
\hline Neutrophils $\left(\times 10^{9} / \mathrm{L}\right)$ (Mean) & 6.2 & 6.0 & 0.82 \\
\hline Lymphocytes (× 109/L) (Mean) & 0.9 & 1.0 & 0.96 \\
\hline Hemoglobin (g/dL) (Mean) & 14.6 & 14.9 & 0.84 \\
\hline Platelets $\left(10^{9} / \mathrm{L}\right)$ (Mean) & 220 & 224 & 0.90 \\
\hline Lactate (mmol/L) (Mean) & 1.7 & 1.0 & 0.39 \\
\hline Creatinine (mg/dL) (Mean) & 0.91 & 0.89 & 0.62 \\
\hline Albumin (g/dL) & 3.5 & 3.6 & 0.46 \\
\hline 25-0H-Vitamine D (ng/dL) (Mean) & 14.9 & 15.5 & 0.31 \\
\hline LDH (UI/L) (Mean) & 386 & 284 & 0.01 \\
\hline D Dimer (mcg/L) (Mean) & 888 & 766 & 0.35 \\
\hline Procalcitonin (ng/mL) (Mean) & 0.25 & 0.13 & 0.37 \\
\hline $\mathrm{C}$ reactive protein (mg/L) (Mean) & 13.2 & 10.5 & 0.66 \\
\hline Ferritin (mcg/L) (Mean) & 872 & 741 & 0.18 \\
\hline IL-6 (pg/mL) (Mean) & 101 & 51 & 0.08 \\
\hline PNR (Mean) & 6.91 & 6.35 & 0.78 \\
\hline LPR (Mean) & 187 & 229 & 0.55 \\
\hline
\end{tabular}

For normal distribution mean and standard deviation were used and for free distribution the median and interquartile range

population did not translate into a higher rate of statistically significant complications. The previous reports in patients with pulmonary infections have shown a correlation with the increase of LDH and elevation in respiratory complications, such as the use of mechanical and non-mechanical ventilation. This is even more prevalent in patients with opportunistic infections or infections by mycobacterium ${ }^{5}$. In the AH1N1 Influenza epidemic of $2009,77.8 \%$ of patients with LDH levels $>225 \mathrm{U} / \mathrm{L}$ had a significant increment in mortality levels ${ }^{17}$.
While comparing clinical outcomes based on biomarkers, we show the relationship that the increment of LDH and age as factors of poor prognosis and as predictors of complications in hospitalized patients. It has been demonstrated that there is a 6 -time increment in the probability of presenting more severe forms of SARC-COV 2 with LDH levels above the standard cutoff value, these patients are 16 times more likely to die from the disease ${ }^{18}$, with a sensibility of up to $100 \%$ and a specificity of $86.67 \%$ for LDH levels higher than $283 \mathrm{U} / \mathrm{L}^{18-20}$. It was of critical importance to evaluate 

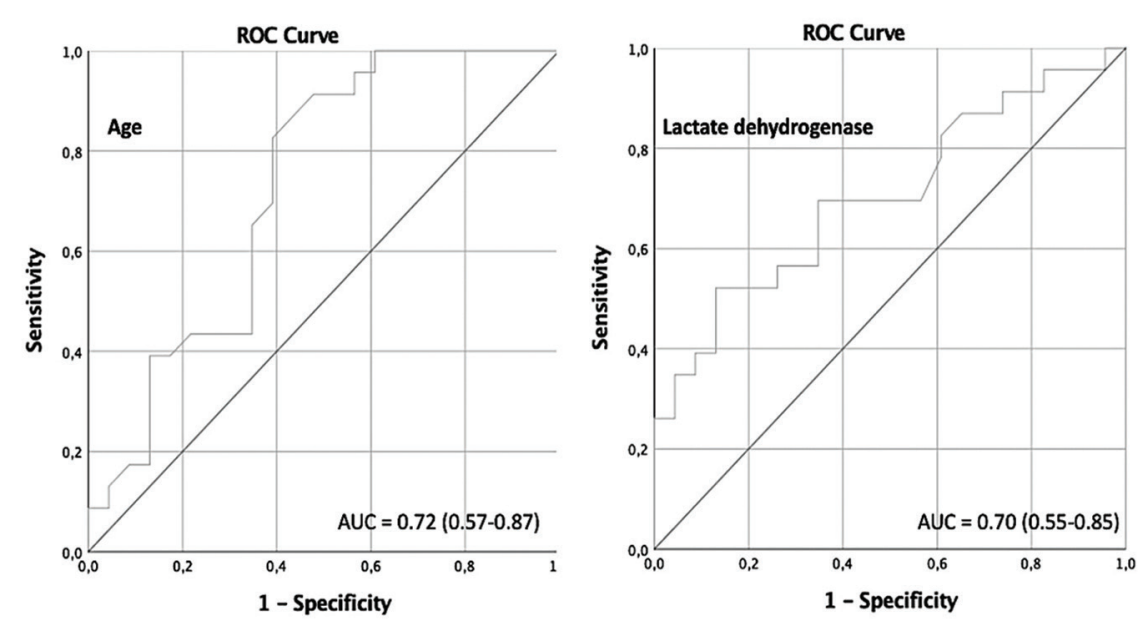

Figure 1. Receiver operating characteristic curve comprising age, glucose, and lactic dehydrogenase as markers of primary outcome.

Table 3. Relative risk for death, use of vasoactive amines, or use of mechanical ventilation

\begin{tabular}{|l|c|c|c|}
\hline Variable & Relative risk & Interval & p value \\
\hline Age $>50$ years & 4.5 & $1.2-16.9$ & 0.003 \\
\hline Glucose $>126 \mathrm{mg} / \mathrm{dL}$ & 2.75 & $1.64-4.58$ & 0.001 \\
\hline Albumin $<3.5 \mathrm{~g} / \mathrm{dL}$ & 1.25 & $0.6-2.2$ & 0.46 \\
\hline Procalcitonin $>2$ (ng/mL) & 0.71 & $0.2-1.8$ & 0.69 \\
\hline $\begin{array}{l}\text { C reactive protein } \\
>\text { (5mg/L) }\end{array}$ & 1.31 & $0.58-2.99$ & 0.72 \\
\hline Ferritin $>1500$ (mcg/L) & 1.82 & $1.08-3.05$ & 0.09 \\
\hline D Dimer $>1000(\mathrm{mcg} / \mathrm{L})$ & 1.19 & $0.67-2.12$ & 0.54 \\
\hline LDH $>410(\mathrm{UI} / \mathrm{L})$ & 2.04 & $1.25-3.34$ & 0.03 \\
\hline IL-6 $>50(\mathrm{pg} / \mathrm{mL})$ & 1.21 & $0.63-2.33$ & 0.53 \\
\hline LNR $>5$ & 1.0 & $0.54-1.8$ & 1.0 \\
\hline LNR $>3$ & 2.2 & $0.64-7.59$ & 0.12 \\
\hline LPR $>300$ & 1,35 & $0.76-2.39$ & 0.51 \\
\hline LPR $>150$ & 0.73 & $0.41-1.30$ & 0.32 \\
\hline
\end{tabular}

these findings and their applicability in our population, as they have demographically distinct characteristics. This was reproduced in our study; therefore, we must deduce that patients with these variables must be monitored in a closer fashion ${ }^{20}$. It is also relevant to note that there exist multiple factors that may elevate levels of $\mathrm{LDH}$; thus, this variable must be considered in the individual context of the patient to be able to use it to establish a prognostic profile. Glucose level was useful to predict disease progression not considering if the patients were previously diabetic and above other biomarkers, this result is consistent with other cohorts ${ }^{21}$. Biomarkers such as IL-6, the NLR, and the LPR did not demonstrate statistical significance in our analysis toward the creation of a prognostic profile and the demonstration of an increment in mortality nor complications. Ferritin levels above 1500 tend to be significant, like other studies, it is possible this may be related to the size of the study. Recent reports have demonstrated a direct relationship between age and an increment in morbidity and mortality rates ${ }^{14,22}$. This correlates directly with our study, showing that patients over 50 years of age were found to have a higher risk of complications included in the primary outcome, such as mechanical ventilation and death. This was not reproduced in patients with hypertension, type two diabetes mellitus, obesity, chronic renal, or hepatic disease. This also may be explained by the sample size and the small number of patients with these comorbidities seen in the ABC Medical Center, as well as how, in general, the patients in our population who have these diseases maintain them under control with medical treatment, which lowers the possibility of presenting complications in itself.

The limitations of our study are: a small sample size, the evolution days before hospital admission were not taken into account, the biomarkers were analyzed in a single moment, an analysis by treatment type was not performed, and the design does not include a comparative arm with pneumonia due to other etiologies. 
The use of biomarkers, specifically as a prognostic profile is of increasing utility in daily clinical practice as a way to predict the evolution of an ill patient and enable intervention in a timely fashion to avoid complications. This is particularly important in a country with limited resources, both in relation to the attention critically ill patients receive and medications that are available to them. To be able to predict complications in patients may also help in relieving health related costs. This study permits the identification of biomarkers to predict such complications and opens a pathway onto a new generation of hypothesis which may be of use toward medical response in this pandemic.

\section{Conclusions}

Patients with SARS-COV 2 severe infection, age over 50 years, glucose above $126 \mathrm{mg} / \mathrm{dl}$, and LDH level above $410 \mathrm{IU}$ were associated with disease progression (death, mechanical, and non-mechanical ventilation or use of vasoactive amines). Ferritin levels above 1500 tend to be significant. Other inflammatory parameters such as NLR and LPR did not show a statistical significant association as predictors of severe disease. Conflicts of interest

The authors declare that does not exist any conflicts of interest

\section{Funding}

The present investigation has not received specific aid from agencies of the public sector, commercial sector, or non-profit entities.

\section{Ethical disclosures}

Protection of human and animal subjects. The authors declare that no experiments were performed on humans or animals for this study.

Confidentiality of data. The authors declare that they have followed the protocols of their work center on the publication of patient data.
Right to privacy and informed consent. The authors declare that no patient data appear in this article.

\section{References}

1. Chan JF, Yuan S, Kok KH, To KK, Chu H, Yang J, et al. A familial cluster of pneumonia associated with the 2019 novel coronavirus indicating person-to-person transmission: a study of a family cluster. Lancet. 2020;395:514-23.

2. Zhu N, Zhang D, Wang W, Li X, Yang B, Song J, et al. China novel coronavirus investigating and research team (2020). A novel coronavirus from patients with pneumonia in China, 2019. N Engl J Med. 2020;382:727-33.

3. Sutton D, Fuchs K, D'Alton M, Goffman D. Universal screening for SARSCoV-2 in women admitted for delivery. N Engl J Med. 2020;382:2163-4.

4. Sakurai A, Sasaki T, Kato S, Hayashi M, Tsuzuki SI, Ishihara T, et al. Natural history of asymptomatic SARS-CoV-2 infection. N Engl J Med. 2020;383:885-6.

5. Huang C, Wang Y, Li X, Ren L, Zhao J, Hu Y, et al. Clinical features of patients infected with 2019 novel coronavirus in Wuhan, China. Lancet. 2020;395:497-506.

6. Petrilli CM, Jones SA, Yang J, Rajagopalan H, O'Donnell L, Chernyak Y, et al. Factors associated with hospital admission and critical illness among 5279 people with coronavirus disease 2019 in New York City: prospective cohort study. BMJ. 2020;369:m1966.

7. Zhang H, Zhou P, Wei Y, Yue H, Wang Y, Hu M, et al. Histopathologic changes and SARS-CoV-2 immunostaining in the lung of a patient with COVID-19. Ann. Int Med. 2020;172:629-32.

8. Tay MZ, Poh CM, Rénia L, MacAry PA, Ng L. The trinity of COVID-19: immunity, inflammation and intervention. Nat Rev Immunol. 2020;20:363-74.

9. Chen IY, Moriyama M, Chang MF, Ichinohe T. Severe acute respiratory syndrome coronavirus viroporin 3a activates the NLRP3 inflammasome. Front Microbiol. 2019;10:50.

10. Zeng F, Huang Y, Guo Y, Yin M, Chen X, Xiao L, et al. Association of inflammatory markers with the severity of COVID-19: a meta-analysis. Int J Infect Dis. 2020;96:467-74.

11. Lagunas-Rangel FA. Neutrophil-to-lymphocyte ratio and lymphocyte-to-C-reactive protein ratio in patients with severe coronavirus disease 2019 (COVID-19): a meta-analysis. J Med Virol. 2020;92:1733-4.

12. Li Q, Guan X, Wu $P$, Wang $X$, Zhou L, Tong $Y$, et al. Early transmission dynamics in Wuhan, China, of novel coronavirus-infected pneumonia. $\mathrm{N}$ Engl J Med. 2020;382:1199-207.

13. COVID 19 México. CONACYT, Centro Geo, Geo Int, DataLab; 2020. Available from: https://www.coronavirus.gob.mx/datos.

14. Guan WJ, Ni ZY, Hu Y, Liang WH, Ou CQ, He JX, et al. China medical treatment expert group for Covid-19 (2020). Clinical characteristics of coronavirus disease 2019 in China. N Engl J Med. 2020;382:1708-20.

15. Jin JM, Bai P, He W, Wu F, Liu XF, Han DM, et al. Gender differences in patients with COVID-19: focus on severity and mortality. Front Public Health. 2020;8:152.

16. Sharma G, Volgman AS, Michos ED. Sex differences in mortality from COVID-19 pandemic: are men vulnerable and women protected? JACC Case Rep. 2020;2:1407-10.

17. Lee IK, Liu JW, Wang L, Yang KD, Li CC, Eng HL. 2009 pandemic influenza $A$ (H1N1): clinical and laboratory characteristics in pediatric and adult patients and in patients with pulmonary involvement. Influenza Other Respir Viruses. 2012;6:e152-61.

18. Martellucci C, Flacco M, Cappadona R, Bravi F, Mantovani L, et al SARS-CoV-2 pandemic: an overview. Adv Biol Regul. 2020;77:100736.

19. Shi J, Li Y, Zhou X, Zhang Q, Ye X, Wu Z, et al. Lactate dehydrogenase and susceptibility to deterioration of mild COVID-19 patients: a multicenter nested case-control study. BMC Med. 2020;18:168.

20. Henry BM, Aggarwal G, Wong J, Benoit S, Vikse J, Plebani M, et al. Lactate dehydrogenase levels predict coronavirus disease 2019 (COVID-19) severity and mortality: a pooled analysis. Am J Emerg Med. 2020;38:1722-26.

21. Zhu B, Jin S, Wu L, Hu C, Wang Z, Bu L, et al. J-shaped association between fasting blood glucose levels and COVID-19 severity in patients without diabetes. Diabetes Res Clin Pract. 2020:168:108381.

22. Wu C, Chen X, Cai Y, Xia J, Zhou X, Xu S, et al. Risk factors associated with acute respiratory distress syndrome and death in patients with coronavirus disease 2019 pneumonia in Wuhan, China. JAMA Intern Med. 2020;180:934-43. 